\title{
Silencing of Aurora kinase A by RNA interference inhibits tumor growth in human osteosarcoma cells by inducing apoptosis and G2/M cell cycle arrest
}

\author{
ZHENHUAN JIANG $^{1,2 *}$, JIANNONG JIANG ${ }^{2 *}$, HUILIN YANG $^{1}$, ZHIJUN GE $^{3}$, QIANG WANG ${ }^{2}$, \\ LEIYAN ZHANG ${ }^{2}$, CHENGUANG $\mathrm{WU}^{2}$ and JINZHI WANG ${ }^{4}$
}

\begin{abstract}
${ }^{1}$ Department of Orthopaedics, The First Affiliated Hospital of Soochow University, Suzhou, Jiangsu 215006; Departments of ${ }^{2}$ Orthopaedics, and ${ }^{3}$ Anesthesiology, People's Hospital of Yixing City, Yixing, Jiangsu 214200; ${ }^{4}$ Department of Cell Biology, School of Medicine, Soochow University, Suzhou, Jiangsu 215123, P.R. China
\end{abstract}

Received November 15, 2013; Accepted December 24, 2013

DOI: 10.3892/or.2014.2986

\begin{abstract}
The overexpression of Aurora kinase A (AURKA), a member of serine/threonine kinase family, has been observed in various types of human cancers. However, the role of AURKA in osteosarcoma (OS), the most common type of primary malignancy arising from bone, has not been clarified. We used AURKA-specific lentivirus-delivered short hairpin RNA (shRNA) to significantly and sustainably silence the endogenous AURKA expression in human OS cells SAOS-2 and U2OS. We found that AURKA downregulation in OS cells prominently decreased colony formation ability in vitro and tumorigenesis ability in vivo. We further evaluated the effect of AURKA silence on cell viability by MTT assay, cell apoptosis and cell cycle by flow cytometer detection. The results showed that AURKA silence inhibited cell viability by inducing cell apoptosis and G2/M cell cycle arrest in OS cells. Taken together, our findings indicate that AURKA plays a crucial role on OS growth by inhibiting cell apoptosis and propelling cell cycle. Inhibition of AURKA by lentivirusdelivered specific shRNA showed the therapeutic potential in treatment of osteosarcoma.
\end{abstract}

\section{Introduction}

Although osteosarcoma (OS) is a relatively rare cancer, it is the most common type of primary malignant bone cancer (1). OS mainly arises in adolescents and young adults (between the ages of 10 to 25). Almost 6 per million adolescents and 2 per million adults will develop OS. By using modern OS treatment

Correspondence to: Professor Jinzhi Wang, Department of Cell Biology, School of Medicine, Soochow University, Suzhou, Jiangsu 215123, P.R. China

E-mail: zuguodadi@msn.com

*Contributed equally

Key words: osteosarcoma, Aurora kinase A, tumorigenicity, RNA interference, lentivirus strategies that combine surgery, chemotherapy, sometimes radiotherapy, the 5-year survival rate for OS patients is still only $60-70 \%$ (2). Thus, there is an urgent need to explore new cancer management methods to treat OS.

Currently, gene therapy, as a novel cancer therapy, is gaining increasing attention. This therapy works by transferring a nucleic acid regulator, which affects expression of a specific tumor-associated gene to cancer cells, such as short hairpin RNA (shRNA) (3). shRNA expression vector system has been established to generate small interfering RNA (siRNA) in mammalian cells. siRNA plays many important roles in the cellular regulatory mechanism, especially in the RNA interference (RNAi) pathway, where siRNA utilises the cell's inherent machinery (Dicer) to degrade target mRNA and thereby silencing specific gene expression (4). Lentivirus vector has been demonstrated to be a highly effective and stable gene transfer tool (5). Therefore, lentivirus-delivered shRNA is considered to be a hopeful tool for gene therapy, which has been used in clinical trials without prominent sideeffects (6).

Aurora kinase A (AURKA), a member of serine/threonine kinase family, is involved in correct centrosome duplication, mauration and separation, as well as in chromosomal segregation during cellular mitosis (7). Overexpression of AURKA gene is evident in various human cancers, including esophageal squamous cell carcinoma, pancreatic, and ovarian cancer, and is implicated in promoting cell proliferation and suppressing cell apoptosis (8-11). Some studies also show that altered activity of AURKA leads to genomic instability and thus to tumorigenesis $(12,13)$.

In the present study, we used lentivirus-mediated shRNA to silence AURKA expression in human OS cells. Then we examined the effect of AURKA silence on tumor growth and the mechanisms.

\section{Materials and methods}

Cell culture. Human OS cell lines SAOS-2, U2OS, MG-63 and HOS U251 were purchased from the American Type Culture Collection (ATCC; Manassas, VA, USA) and cultured in 
Dulbecco's modified Eagle's medium(DMEM) containing 10\% fetal bovine serum (FBS), $100 \mathrm{U} / \mathrm{ml}$ penicillin and $100 \mu \mathrm{g} / \mathrm{ml}$ streptomycin at $37^{\circ} \mathrm{C}$ under humidified air containing $5 \% \mathrm{CO}_{2}$.

RNA extraction and quantitative real-time RT-PCR. Total RNA was extracted from cells with TRIzol reagent (Invitrogen, Carlsbad, CA, USA) according to the manufacturer's instructions. The cDNA was synthesized using the RevertAid First Strand cDNA Synthesis kit (Fermentas, Vilnius, Lithuania) according to the manufacturer's protocol. Genes expression were detected by quantitative real-time RT-PCR (qRT-PCR) using the standard SYBR-Green RT-PCR kit (Takara, Kyoto, Japan) as recommended by the manufacturer. The specific primer pairs and the amplified products are as follows: AURKA (286 bp), sense: 5'-GCC CTG TCT TAC TGT CAT TCG-3'; antisense: 5'-AGG TCT CTT GGT ATG TGT TTG C-3'; GAPDH (121 bp), sense: 5'-TGA CTT CAA CAG CGA CAC CCA-3'; antisense: 5'-CAC CCT GTT GCT GTA GCC AAA-3'. The relative levels of AURKA gene mRNA transcripts were normalized to the control GAPDH. Relative gene expression was quantified using the GraphPad Prism 5.0 software (GraphPad Software, Inc., San Diego, CA, USA).

AURKA gene silence mediated by lentivirus-delivered shRNA. shRNA candidate target sequences to AURKA is 5'-GAAAG CTCCACATCAATAA-3'. The oligonucleotides encoding the shRNA sequence or non-silencing control (NC) sequence (5'-TTCTCCGAACGTGTCACGT-3') were inserted into the GFP express vector pGCL-GFP (Shanghai GeneChem Co., Ltd., Shanghai, China). NC RNAi was used as a negative RNAi control. The recombinant virus was packaged using the Lentivector Expression systems (Shanghai GeneChem). SAOS-2 and U2OS cells were infected. After 3 days, GFP-positive cells were counted under fluorescence microscope (Olympus, Tokyo, Japan). AURKA expression after infection of shRNA expression construct was revealed by realtime PCR on day 4.

Western blotting. The whole-cell lysates were subjected to SDS-PAGE. The blots were incubated with AURKA antibodies (Cell Signaling Technology, Beverly, MA, USA) and then with anti-rabbit IgG peroxidase-conjugated secondary antibody (Santa Cruz Biotechnology, Santa Cruz, CA, USA) and chemiluminescent substrates. Hybridization with antiGAPDH (Santa Cruz Biotechnology) was used to confirm equal protein loading.

Colony-forming assay. Exponentially growing cells were suspended in complete growth medium and seeded in 6-well plates with 200 cells/well. The plates were maintained at $37^{\circ} \mathrm{C}$ in a humidified incubator with $5 \% \mathrm{CO}_{2}$ for two weeks. The visible colonies were subsequently recorded by a fluorescence microscope. After fixation in paraformaldehyde, the colonies were stained with Giemsa for $10 \mathrm{~min}$ followed by taking images with a digital camera.

Tumorigenesis assay. Male nude mice (7-8 weeks old, weighing 18-20 g) were obtained from the Shanghai Experimental Center, Chinese Science Academy, Shanghai, and maintained at an animal facility under pathogen-free conditions.
All animals received humane care according to the National Research Council's guidelines. The study was approved by the Animal Experimentation Committee of Soochow University.

SAOS-2 cells infected with control lentivirus or AURKAspecific siRNA lentivirus were resuspended in PBS at a final density of $1 \times 10^{7}$ cells $/ \mathrm{ml}$. Each group of nude mice was injected into the axilla with $100 \mu 1$ of the corresponding cell suspension (equivalent to $1 \times 10^{6}$ cells). The mice were sacrificed 3 weeks after injection and examined for tumor growth. The effect of AURKA knockdown on tumor growth was assessed by measuring tumor size and tumor weight. Measurement of tumor size was used to calculate the tumor volume using the formula: $1 / 2 * a * b^{2}$, where $a$ is the major diameter and $b$ is the minor diameter.

Cell viability assay. Cell viability was evaluated by 3-[4,5-dimethylthiazol-2-yl]-2,5-diphenyltetrazolium bromide (MTT) (Sigma Chemical, St. Louis, MO, USA) assay. The test cells in exponential growth were plated at a final concentration of $2 \times 10^{3}$ cells/well in 96-well culture plates for different culture time. Then MTT $(10 \mu \mathrm{l}, 5 \mathrm{mg} / \mathrm{ml})$ was added to each well. Incubation was continued at $37^{\circ} \mathrm{C}$ for $4 \mathrm{~h}$. The reaction was terminated by replacing the MTT-containing medium with $100 \mu \mathrm{l}$ DMSO and the formazan salts were dissolved by gentle shaking for about $10 \mathrm{~min}$ at room temperature. Optical density (OD) of each well was measured at $490 \mathrm{~nm}$ using ELISA reader (ELx808; Bio-Tek Instruments, Winooski, VT USA).

Detection of apoptosis by flow cytometry. Cells were stained with APC labeled Annexin V (eBioscience, San Diego, CA, USA) to detect apoptotic cells (Annexin V positive). A total of $1.0 \times 10^{6}$ cells were washed twice with ice-cold PBS and incubated for $15 \mathrm{~min}$ in $100 \mu \mathrm{l}$ staining buffer including $5 \mu \mathrm{l}$ APC labeled Annexin V. Fluorescence-activated cell sorting (FACS) analysis for Annexin V staining was performed by flow cytometer (FACSCalibur; BD Biosciences, San Jose, CA, USA). All experiments were performed in triplicate.

Cell cycle assay. Cells were seeded into a 6-cm dish, harvested at specific times by centrifuging at $1,200 \mathrm{rpm}$ for $5 \mathrm{~min}$. Following washing with pre-cooled PBS (pH 7.4) twice, cells were fixed in $70 \%$ alcohol. Percentage of cells in each stage of the cell cycle was determined by staining with propidium iodide (PI) (Sigma). The analysis of cell cycle distribution was performed by a flow cytometer (FACSCalibur; BD Biosciences) in accordance with the manufacturer's guidelines.

Statistical methods. Data are expressed as the mean \pm SEM. Statistical significance was determined by the Student's t-test and one-way ANOVA using GraphPad Prism 5.0 software (GraphPad software). A value of $\mathrm{P}<0.05$ was considered to indicate a statistically significant difference.

\section{Results}

Lentivirus-delivered shRNA mediated downregulation of AURKA expression in OS cells. We used RT-PCR to detect AURKA expression in the OS cell line SAOS-2, U2OS, MG-63 and HOS. The results showed that AURKA gene is 
A

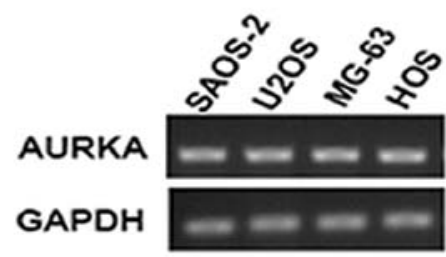

B

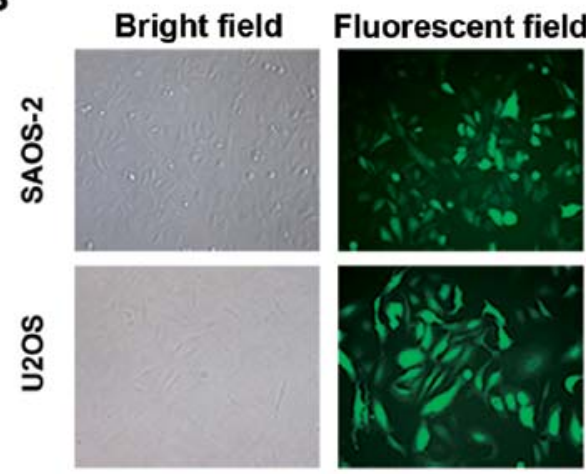

C

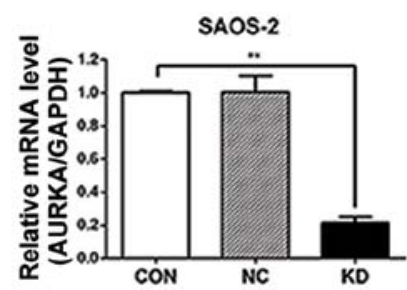

D

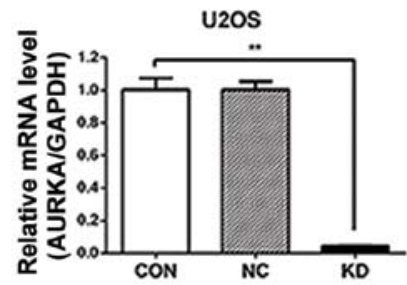

AURKA

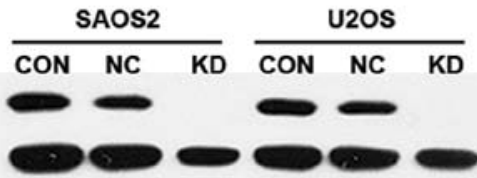

Figure 1. Lentivirus-delivered shRNA stably decreased AURKA expression in human OS cells. (A) AURKA mRNA expression of human OS cell lines SAOS-2, U2OS, MG-63 and HOS were determined by RT-PCR. (B) Transduction efficiency of lentivirus in SAOS-2 and OS cells was estimated 3 days after infection at MOI of 5 (original magnification, $x 200$ ). (C) Total RNA was extracted at 4 days after infection and relative AURKA mRNA expression was determined by qRT-PCR. GAPDH was used to an internal gene. Data represent the mean \pm SEM (n=3; $\left.{ }^{* *} \mathrm{P}<0.01\right)$. (D) AURKA protein level of infected cells were detected by western blot analysis. CON, uninfected cells; NC, cells infected with control lentivirus; KD, cells infected with AURKA-shRNA lentivirus.

expressed in these human OS cell lines (Fig. 1A). To study the role of AURKA in OS cells, we used recombinant lentivirus delivered shRNA to silence AURKA expression in SAOS-2 and U2OS cells. The infection efficiency of AURKA-shRNA lentivirus was $>80 \%$ in both SAOS-2 and U2OS cells. Most of the infected cells expressed GFP protein 3 days after infection (Fig. 1B).

To evaluate the effectiveness of lentivirus-mediated infection on the silencing of AURKA expression, lentivirus infected OS cells were assayed by real-time PCR. The data demonstrated that AURKA mRNA expression was efficiently suppressed in AURKA-shRNA lentivirus infected cells (KD) compared to uninfected cells (CON). There was no significant difference in AURKA mRNA expression between the CON group and control lentivirus infected cells (NC) group (Fig. 1C). Western blot assay also showed that lentivirus-delivered AURKA-shRNA silenced AURKA protein expression in SAOS-2 and U2OS cells (Fig. 1D). These results showed that AURKA gene could be significantly and sustainably downregulated by lentivirus-mediated AURKA-specific shRNA in OS cells.

AURKA silencing in OS cells leads to the decrease of colony formation ability in vitro and tumorigenicity in vivo. Then we explored how AURKA silencing affects tumorigenesis of OS cells in vitro and in vivo. The colony formation assay showed that compared to $\mathrm{CON}$ and $\mathrm{NC}$ groups, KD showed a marked decrease $(\sim 80 \%)$ of the colony number in SAOS-2 cells (Fig. 2). These data suggested that AURKA silencing suppressed the tumorigenic ability of SAOS-2 cells in vitro. We injected the SAOS-2 cells of NC and KD groups into axilla of the nude mice, respectively. Representative pictures
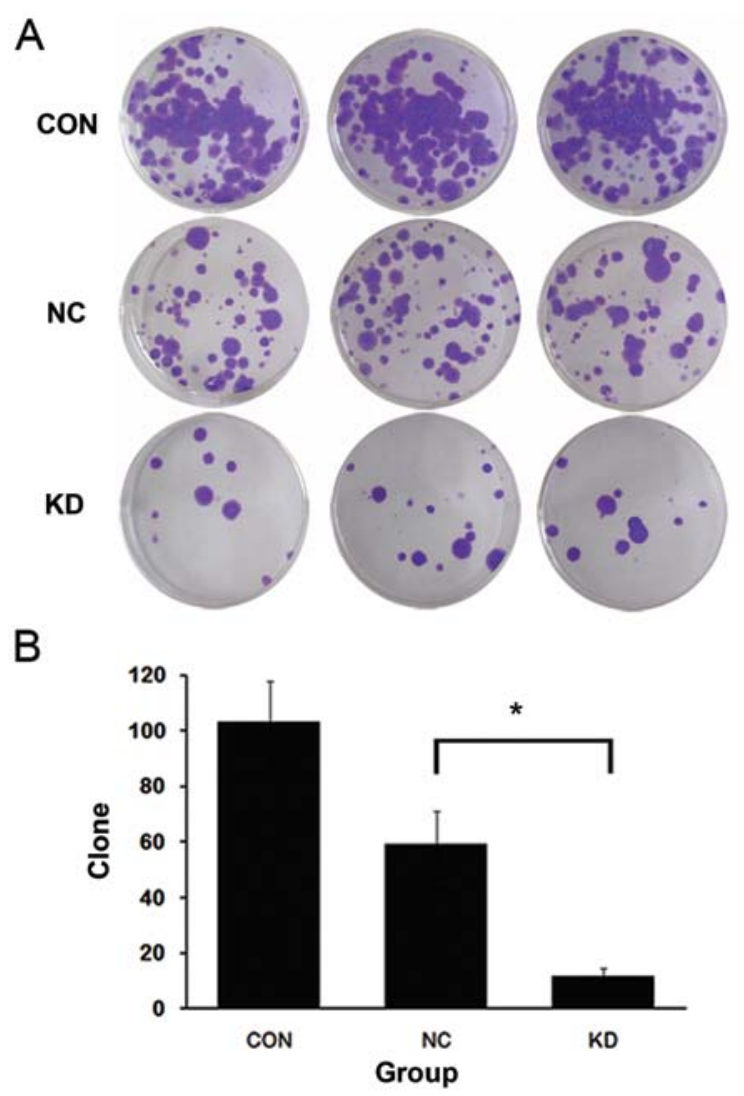

Figure 2. Knockdown of AURKA decreased colony formation ability of OS cells. Cell colony formation assay in SAOS-2 cells. Cells were seeded at 2,000/well and allowed for 14 days to form colonies. (A) The colonies stained with Giemsa were observed under fluorescence. Colony numbers were counted and recorded. (B) Data represent the mean \pm SD of three independent experiments. ${ }^{* *} \mathrm{P}<0.01$. CON, uninfected cells; $\mathrm{NC}$, cells infected with control lentivirus; KD, cells infected with AURKA-shRNA lentivirus. 
A
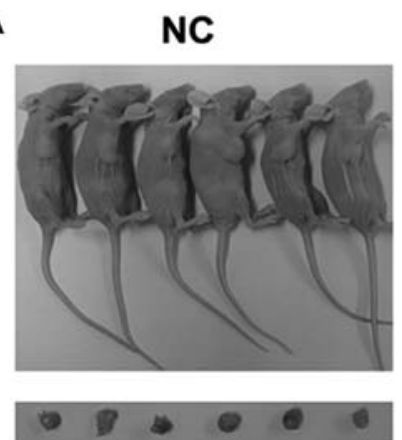

B

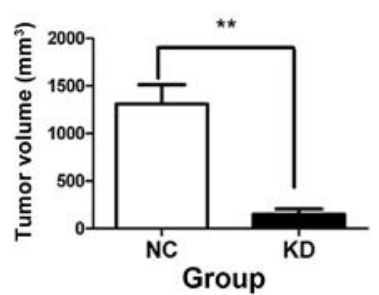

KD

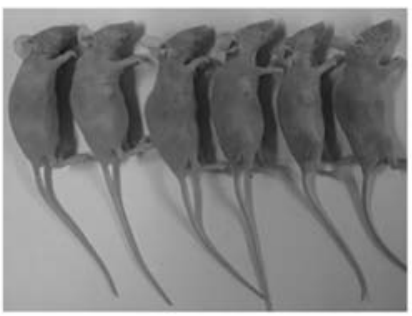

C
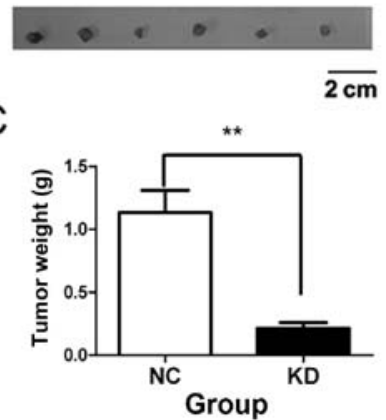

Figure 3. Downregulation of AURKA expression reduced tumorigenicity in OS cells. (A) NC and KD groups of nude mice were injected the corresponding infected SAOS- 2 cells $\left(10^{6}\right.$ cells/mice $)$ into the axilla. After 3 weeks, the mice were sacrificed and the tumors harvested. (B and C) Quantitative measurement of (B) tumor volume and (C) weight. Data represent the mean $\pm \mathrm{SD}$ of each group $\left(\mathrm{n}=6 ;{ }^{* *} \mathrm{P}<0.01\right)$.

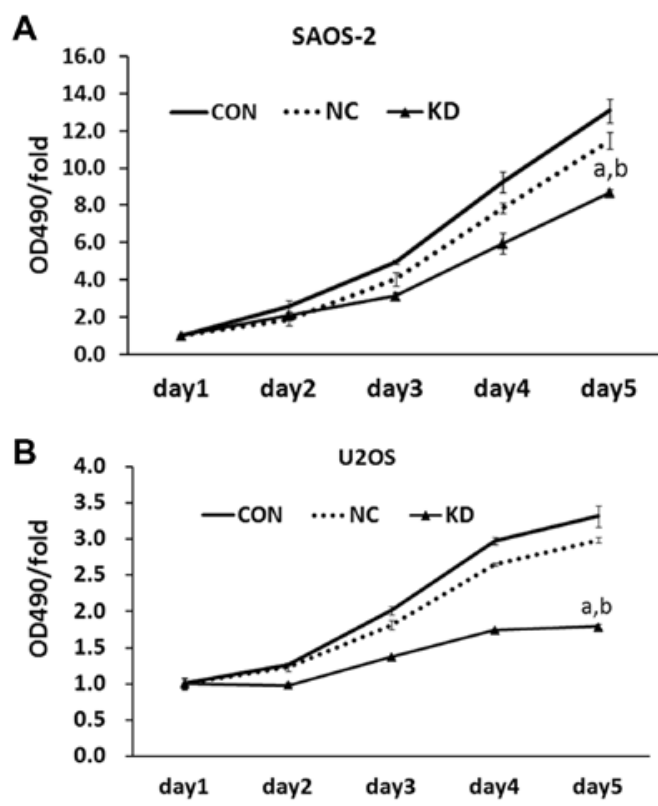

Figure 4. AURKA-specific shRNA reduced cell viability in OS cells. Cell viability curves of three groups during 5-day evaluation by MTT assay in (A) SAOS-2 and (B) U2OS cells. Data represent the means \pm SEM $\left(n=3 ;{ }^{a} \mathrm{P}<0.05\right.$ vs. $\mathrm{CON}$; ${ }^{\mathrm{P}} \mathrm{P}<0.05$ vs. $\mathrm{NC}$ ). $\mathrm{CON}$, uninfected cells; $\mathrm{NC}$, cells infected with control lentivirus; KD, cells infected with AURKA-shRNA lentivirus.

of tumors at 3 weeks after cell injection are shown in Fig. 3A. Compared to $\mathrm{NC}$ group, the mean tumor volume and weight of KD group were reduced by 90 and 80\%, respectively (Fig. 3B and C). These data demonstrated that AURKA silence significantly inhibits colony formation ability and tumorigenicity of SAOS-2 cells.

AURKA silence induces cell apoptosis and cell cycle arrest at $G 2 / M$ phase in $O S$ cells. We attempted to explore why AURKA silence affected tumorigenesis of human OS cells. MTT assay showed that AURKA silence decreased cell viability of SAOS-2 cells in a time-dependent manner. Following a 5-day period, cell viability of KD group was only $66 \%$ of that of CON group, whereas control lentivirus had no effect on the viability of SAOS-2 cells (Fig. 4A). The data in U2OS cells demonstrated a similar result (Fig. 4B). This showed that AURKA silencing remarkably inhibits cell viability in OS cells.

We further investigated how AURKA silence inhibits the viability of OS cells. We used Annexin V-APC staining and FACS analysis to determine whether the downregulation of AURKA gene induces OS cell apoptosis. The data indicated that KD group had more apoptotic cells (Annexin $\mathrm{V}$ positive) compared to those of $\mathrm{CON}$ and $\mathrm{NC}$ group (Fig. 5A and $\mathrm{B}$ ). We used PI staining and FACS analysis to identify AURKAshRNA lentivirus-induced changes in the cell cycle of OS cells. The data showed that downregulation of AURKA expression led to the percentage of SAOS-2 cells in G0/G1 phase significantly decreased from $46.85 \%$ in NC group to $37.5 \%$ in KD group, and the percentage of SAOS-2 cells in G2/M phase prominently increased from $13.48 \%$ in NC group to $26.49 \%$ in KD group. There were no significant difference in cell cycle distribution between CON group and NC group (Fig. 6A). The study in U2OS cells revealed similar results (Fig. 6B). In view of the decrease of cell viability, these data suggested that AURKA silence caused an arrest in G2/M phase, but no more proliferative activity. The above results showed that AURKA silence suppressed cell viability by inducing cell apoptosis and cell cycle arrest at G2/M phase in OS cells.

\section{Discussion}

Surgery remains the main option for OS patients. However, even with the advanced techniques available at present, the rate of local recurrence in non-metastatic OS patients is still as high as $46 \%$ (14). Other conventional OS treatments, including chemotherapy and radiotherapy, are mainly palliative alternatives for cases where surgery is not feasible. Both chemotherapy and radiotherapy have disadvantages, as it is not possible to differentiate between normal and cancerous cells, leading to destruction of cancerous as well as normal cells. Therefore, novel therapeutic approaches are urgently required.

Overexpression of oncogenes and upregulation of their associated signaling transduction pathways are known as the driving forces in OS progression (15). RNAi is an effective method for silencing specific genes (16). Compared to conventional targeting gene technologies including chemical inhibitors, ribozymes and deoxyribozymes, which often cause side-effects, shRNA are more specific inhibitors of gene expression with less toxicity, and can be relatively easily infected into cancer cells by lentivirus delivery (17). Hence, lentivirus-delivered shRNA mediated gene silencing has significant therapeutic potential for cancer.

In the present study, our data suggested that AURKAshRNA lentivirus leads to efficient and specific inhibition of endogenous AURKA mRNA expression in human OS 
A
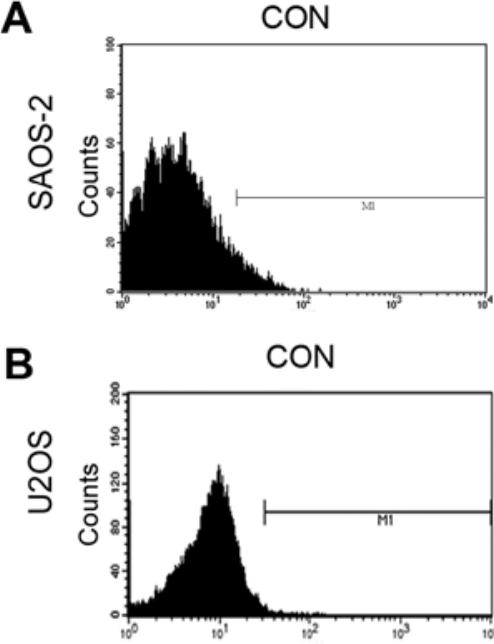

NC

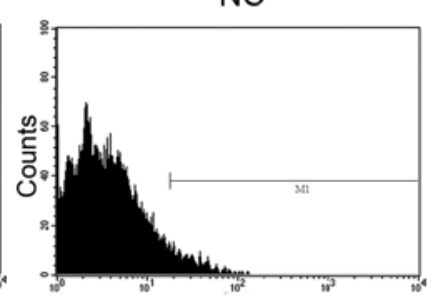

NC

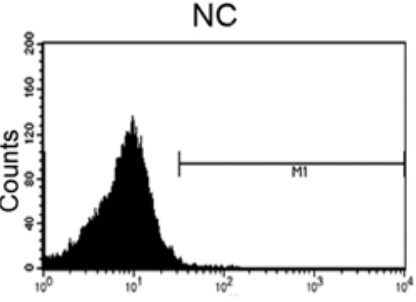

KD

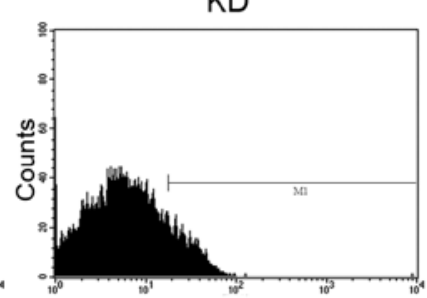

$\mathrm{KD}$

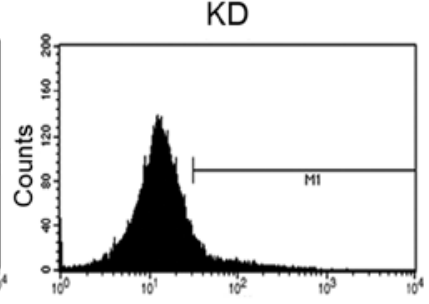

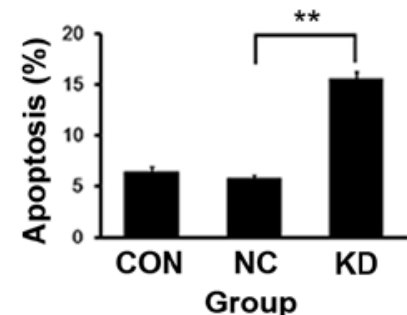

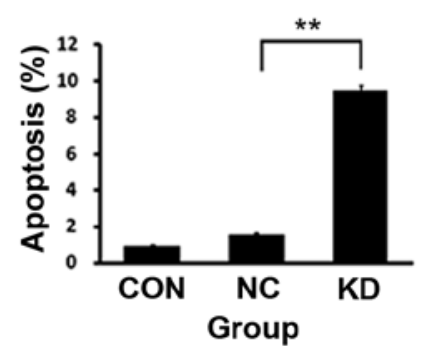

Figure 5. AURKA-specific shRNA induced cell apoptosis in OS cells. Cell apoptosis was detected Annexin V-APC staining and FACS analysis in (A) SAOS-2 and (B) U2OS cells. The percentages of apoptotic (Annexin V positive) cells represent the mean $\pm \mathrm{SD}$ of three independent experiments (right). ${ }^{* *} \mathrm{P}<0.01$. CON, uninfected cells; NC, cells infected with control lentivirus; KD, cells infected with AURKA-shRNA lentivirus.

A

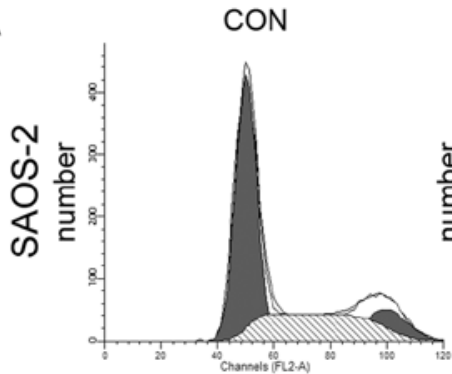

B

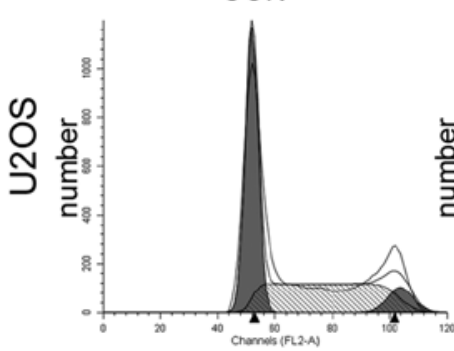

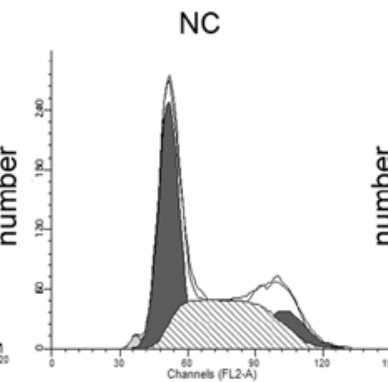

$\mathrm{NC}$

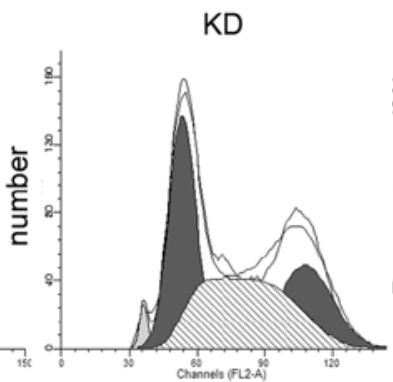

KD
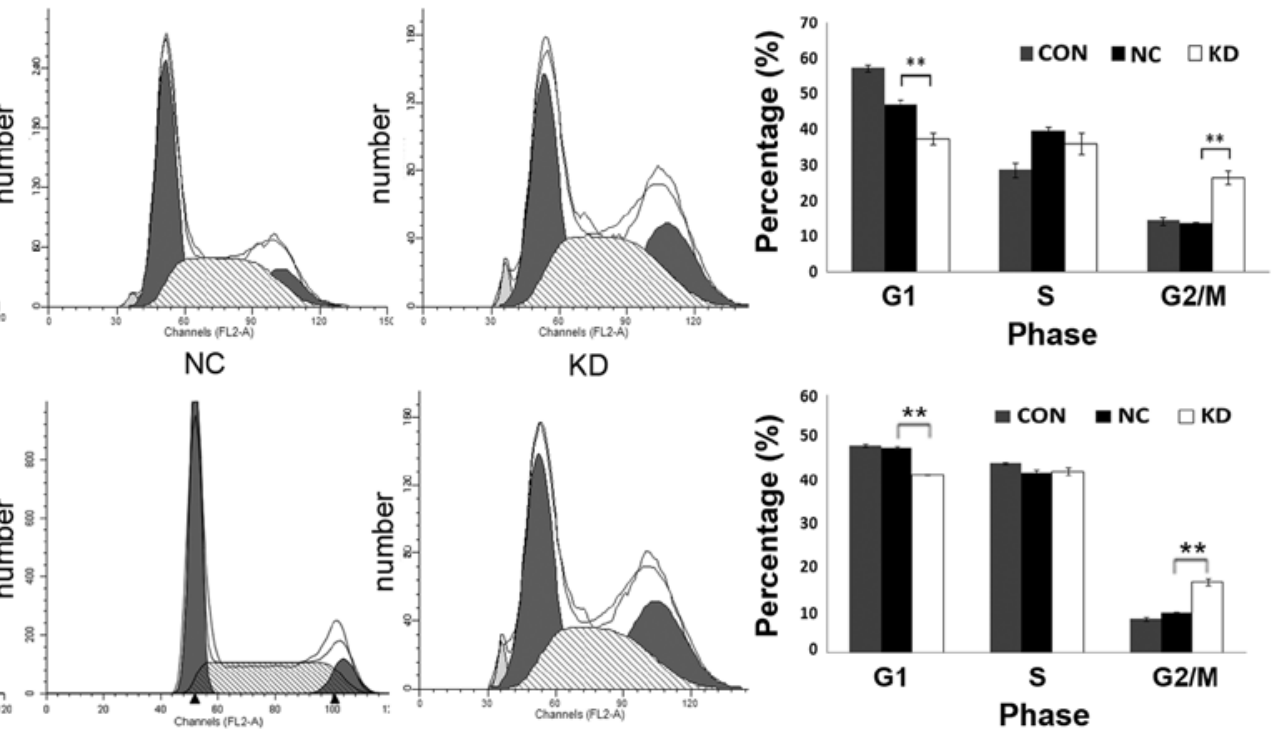

Figure 6. AURKA-specific shRNA induced cell cycle arrest in OS cells. Cell cycle analysis of three cell groups was detected by PI staining and FACS analysis in (A) SAOS-2 and (B) U2OS cells. The percentages of cell cycle phase represent the means \pm SD of three independent experiments (right). ${ }^{* *} \mathrm{P}<0.01$. CON, uninfected cells; NC, cells infected with control lentivirus; KD, cells infected with AURKA-shRNA lentivirus.

cells. AURKA-shRNA lentivirus infected SAOS-2 cells prominently decreased colony-forming ability in vitro and tumorigenicity in vivo. AURKA silence showed a significant decrease of cell viability and a cell cycle arrest in $\mathrm{G} 2 / \mathrm{M}$ phase in OS cells. As reported previously, AURKA is localized mainly at spindle poles and the mitotic spindle during mitosis, where it regulates the functions of centrosomes, spindles and kinetochores required for proper mitotic progression (18). Overexpression of AURKA contributes to genetic instability and tumorigenesis by disrupting the proper assembly of the mitotic checkpoint complex at the level of the Cdc20-BubR1 interaction (19). Moreover, AURKA is a key regulatory component of the p53 pathway, as its overexpression leads to increased p53 degradation, thus facilitating oncogenic transformation (20). These characteristics provide an explanation for the decrease of cell viability and tumorigenicity of AURKA-shRNA infected cells. Moreover, although AURKA is also expressed in normal cells during mitosis, its expression level in normal tissue is quite low. Therefore, AURKA is an ideal target for shRNA-mediated gene cancer therapy.

In summary, our studies investigated and confirmed the significant effect of downregulation of AURKA expression in human OS cells, showing that AURKA silence can induce cell apoptosis and cell arrest in G2/M phase of the cell cycle, and, thus, inhibits colony formation ability and tumorigenicity of OS cells. Furthermore, the present study exhibited 
the feasibility of AURKA-targeting gene therapy for OS, which provided an important theoretical direction for clinical practice of OS.

\section{Acknowledgements}

The present study was supported by a grant from the National Natural Science Foundation of China (no. 81172347). The authors thank Dr Wenxiang Wei (Soochow University, Suzhou, China) for his sincere help and technical support.

\section{References}

1. Dorfman HD and Czerniak B: Bone cancers. Cancer 75: 203-210, 1995.

2. Bielack SS, Kempf-Bielack B, Delling G, et al: Prognostic factors in high-grade osteosarcoma of the extremities or trunk: an analysis of 1,702 patients treated on neoadjuvant cooperative osteosarcoma study group protocols. J Clin Oncol 20: 776-790, 2002.

3. Culver KW, Anderson WF and Blaese RM: Lymphocyte gene therapy. Hum Gene Ther 2: 107-109, 1991.

4. Abdelrahim M, Safe S, Baker C and Abudayyeh A: RNAi and cancer: implications and applications. J RNAi Gene Silencing 2: 136-145, 2006.

5. Rubinson DA, Dillon CP, Kwiatkowski AV, et al: A lentivirusbased system to functionally silence genes in primary mammalian cells, stem cells and transgenic mice by RNA interference. Nat Genet 33: 401-406, 2003.

6. Manilla P, Rebello T, Afable C, et al: Regulatory considerations for novel gene therapy products: a review of the process leading to the first clinical lentiviral vector. Hum Gene Ther 16: 17-25, 2005.

7. Dutertre S, Descamps S and Prigent C: On the role of aurora-A in centrosome function. Oncogene 21: 6175-6183, 2002.

8. Tong T, Zhong Y, Kong J, et al: Overexpression of Aurora-A contributes to malignant development of human esophageal squamous cell carcinoma. Clin Cancer Res 10: 7304-7310, 2004.
9. Tanaka E, Hashimoto Y, Ito T, et al: The clinical significance of Aurora-A/STK15/BTAK expression in human esophageal squamous cell carcinoma. Clin Cancer Res 11: 1827-1834, 2005.

10. Li D, Zhu J, Firozi PF, et al: Overexpression of oncogenic STK15/ BTAK/Aurora A kinase in human pancreatic cancer. Clin Cancer Res 9: 991-997, 2003.

11. Gritsko TM, Coppola D, Paciga JE, et al: Activation and overexpression of centrosome kinase BTAK/Aurora-A in human ovarian cancer. Clin Cancer Res 9: 1420-1426, 2003.

12. Miyoshi Y, Iwao K, Egawa C and Noguchi S: Association of centrosomal kinase STK15/BTAK mRNA expression with chromosomal instability in human breast cancers. Int J Cancer 92: 370-373, 2001.

13. Sen S, Zhou H, Zhang RD, et al: Amplification/overexpression of a mitotic kinase gene in human bladder cancer. J Natl Cancer Inst 94: 1320-1329, 2002.

14. Bacci G, Ferrari S, Mercuri M, et al: Neoadjuvant chemotherapy for osteosarcoma of the extremities in patients aged 41-60 years: outcome in 34 cases treated with adriamycin, cisplatinum and ifosfamide between 1984 and 1999. Acta Orthop 78: 377-384, 2007.

15. Murdoch D and Sager J: Will targeted therapy hold its promise? an evidence-based review. Curr Opin Oncol 20: 104-111, 2008.

16. Li CX, Parker A, Menocal E, Xiang S, Borodyansky L and Fruehauf JH: Delivery of RNA interference. Cell Cycle 5: 2103-2109, 2006.

17. Dave RS and Pomerantz RJ: RNA interference: on the road to an alternate therapeutic strategy! Rev Med Virol 13: 373-385, 2003.

18. Marumoto T, Zhang D and Saya H: Aurora-A - a guardian of poles. Nat Rev Cancer 5: 42-50, 2005.

19. Ke YW, Dou Z, Zhang J and Yao XB: Function and regulation of Aurora/Ipllp kinase family in cell division. Cell Res 13: 69-81, 2003.

20. Katayama H, Sasai K, Kawai H, et al: Phosphorylation by Aurora kinase A induces Mdm2-mediated destabilization and inhibition of p53. Nat Genet 36: 55-62, 2004. 